\title{
Regularity for a Variational Inequality with a Pseudodifferential Operator of Negative Order
}

\author{
R. Schumann
}

\begin{abstract}
We prove that the solution of a variational inequality on a submanifold in $\mathbb{R}^{n}$ involving a pseudodifferential operator of order -1 is bounded.
\end{abstract}

Keywords: Variational inequalities, regularity of solutions, pseudodifferential operators AMS subject classiflcation: 49 A 29, 47 G 05, 47 H 05

\section{Introduction}

Consider the variational inequality to find $u \in K$ such that $(v-u, A u) \geq(v-u, b)$ for all $v \in K$, where $b \in W^{\frac{1}{2}, 2}(S)$ is given, $K$ denotes the positive cone of the Hilbert space $W^{-\frac{1}{2}, 2}(S)$ and $A$ is an elliptic pseudodifferential operator of the negative order -1 on a closed manifold $S \subset \mathbb{R}^{n}$.

Variational inequalities are nonlinear problems even if the operator $A$ is linear because $K$ fails to be a linear subspace of $W^{-\frac{1}{2}, 2}(S)$. The usual setting is that $A$ maps a Banach or Hilbert space $X$ into its dual $X^{*}$. In many applications $X$ is a Sobolev space and $A$ denotes a linear elliptic differential operator of order $m$. By energetic considerations, for example, it is often easy to prove the (weak) solvability of the variational inequality. Concerning the regularity of weak solutions we find two different situations: For elliptic equations $A u=b$ the inclusion $b \in W^{k, 2}$ implies, in general, the inclusion $u \in W^{k+m, 2}$. In contrast to this case, problems for variational inequalities have limited regularity, i.e. even if $b$ is smooth, their solutions $u$ cannot overcome a certain threshold of smoothness. For instance, Shamir [14] gave an example where $u \notin W^{3,2}(\Omega) \cup W^{2,4}(\Omega)$ for $A=-\Delta+I, b \in W^{1, p}$ for all $p>1$ and $K=\left\{u \in W^{1,2}(\Omega): u \geq 0\right.$ on $\left.\Gamma \subset \partial \Omega\right\}$ (cf. Lions [9: Section 8.2] and Rodrigues [12:'p. 279]). For variational inequalities with elliptic differential operators the regularity of solutions was investigated, e.g., by Kinderlehrer [6], Kinderlehrer and Stampacchia [8], and Uralzeva [2, 17]. The case of systems of variational inequalities with one-sided obstacles was treated in the papers of Kinderlehrer [7] (systems in $\mathbb{R}^{2}$ ) and Schumann [13] (Lamé's system of elasticity in $\mathbb{R}^{N}(N \geq 2)$.

It seems however that problems concerning regularity of solutions of variational inequalities have not been considered if the operator $A$ is a pseudodifferential operator

R. Schumann: Universität Leipzig, Institut für Mathematik, Augustuspl. 10, D - 04109 Leipzig 
of negative order. This case can also be motivated by a physical example (see [10]). A-priori the solution $u$ of the variational inequality only belongs to the Sobolev space $W^{-\frac{1}{2}, 2}(S)$ of negative order $-\frac{1}{2}$. Thus we are interested to prove more regularity for the solution. In Section 5 we shall prove the following result.

Theorem. Suppose $b \in W^{\gamma, r}(S)$ for some $\gamma \in(1,2)$ and $r>\frac{N}{\gamma_{-1}}$. Then the solution $u \in K$ of the variational inequality (1) below is essentially bounded, i.e. $u \in$ $L_{\infty}(S)$.

We use the following notation. The norm in the Lebesgue space $L_{p}(U)$ where $U \subset \mathbb{R}^{n}$ denotes an open set is

$$
\|u\|_{p}=\|u\|_{p, U}=\left(\int_{U}|u(x)|^{p} d x\right)^{1 / p}
$$

and

$$
\|u\|_{\gamma, p}=\left(\|u\|_{p}^{p}+|u|_{\gamma, p}^{p}\right)^{1 / p}
$$

denotes the norm in the Sobolev space $W^{\gamma, p}(U)$ with $\gamma \in(0,1)$ where the seminorm $|u|_{\gamma, p}$ is defined by

$$
|u|_{\gamma, p}=\left(\iint_{U \times U}|x-y|^{-N-\gamma p}|u(x)-u(y)|^{p} d x d y\right)^{1 / p}
$$

The set of pseudodifferential operators of order $m$ acting on $U$ is denoted by $\Psi^{m}(U)$.

\section{Problem and approximation (I)}

We suppose that $S$ is a smooth compact $N$-dimensional manifold $(N \geq 2)$ without boundary $(\partial S=\emptyset)$. Consider the following variational inequality:

Find $u \in K$ such that

$$
(v-u, A u) \geq(v-u, b) \quad \text { for all } v \in K
$$

where $b \in W^{\frac{1}{2}, 2}(S)$ is given and $K$ is the positive cone of the Hilbert.space $W^{-\frac{1}{2}, 2}(S)$, i.e.

$$
K=\left\{v \in W^{-\frac{1}{2}, 2}(S):(v, \varphi) \geq 0 \text { for all } \varphi \in \mathcal{D}(S) \text { such that } \varphi \geq 0 \text { on } S\right\} \text {. }
$$

Clearly $K$ is a closed cone of the Sobolev space $X=W^{-\frac{1}{2}, 2}(S)$. We denote the norm in $X$ by $\|\cdot\|_{-\frac{1}{2}, 2}$ and make the following hypotheses on the linear continuous operator $A: W^{-\frac{1}{2}, 2}(S) \rightarrow W^{\frac{1}{2}, 2}(S):$

(H1) There exists a constant $c>0$ such that $(v, A v) \geq c\|v\|_{-\frac{1}{2}, 2}^{2}$ for all $v \in X$. 
(H2) For sake of technical simplicity, we assume that a part $\Gamma$ of $S$ lies in the hyperplane $\mathbb{R}^{N} \subset \mathbb{R}^{n}(n=N+1)$. Furthermore we suppose that the principal symbol of the pseudodifferential operator $A \in \Psi^{-1}(S)$ on $\Gamma$ is given by

$$
\sigma_{-1}(A)\left(x^{\prime}, \xi^{\prime}\right)=\left|\xi^{\prime}\right|^{-1} \quad \text { for }\left(x^{\prime}, 0\right) \in \Gamma
$$

where $x^{\prime}=\left(x_{1}, \ldots, x_{N}\right)$ and $\xi^{\prime}=\left(\xi_{1}, \ldots, \xi_{N}\right)$ (the general case can be handled after a coordinate transform).

It follows from hypothesis (H1) that the variational inequality (1) has a unique solution $u \in K$ (for a proof cf. Lions [9: Chapter 2.8.2/Theorem 8.1]). Hypothesis (H2) will be used in Sections 4 and 5 to prove regularity of the solution.

To prove regularity we first approximate the solution $u$ of variational inequality (1) by solutions $u^{\delta}(\delta>0)$ of the following family of variational inequalities:

Find $u^{\delta} \in K_{1}$ such that

$$
\delta\left(v-u^{\delta} \mid u^{\delta}\right)+\left(v-u^{\delta}, A u^{\delta}\right) \geq\left(v-u^{\delta}, b^{\delta}\right) \quad \text { for all } v \in K_{1}
$$

where

$$
K_{1}=K \cap L_{2}(S)=\left\{v \in L_{2}(S): v(x) \geq 0 \text { a.e. on } S\right\},
$$

$b^{\delta} \in W^{\frac{1}{2}, 2}$ and $(\cdot \mid \cdot)$ denotes the inner product in $L_{2}(S)$.

We will show that the family $\left(u^{\delta}\right)_{\delta>0}$ of solutions of variational inequalities (4) approximates the solution $u$ of variational inequality (1).

Proposition 1. Let $b, b^{\delta} \in W^{\frac{1}{2}, 2}(S)$. Then the following assertions are true.

1. For any $\delta>0$, there exists a unique solution $u^{\delta} \in K_{1}$ of inequality (4).

2. If $\sup _{\delta}\left\|b^{\delta}\right\|_{\frac{1}{2}, 2}<+\infty$, then $\sup _{\delta}\left\|u^{\delta}\right\|_{-\frac{1}{2}, 2}<+\infty$.

3. If $b^{\delta} \rightarrow b$ in $W^{\frac{1}{2}, 2}(S)$ as $\delta \rightarrow+0$, then $u^{\delta} \rightarrow u$ in $X=W^{-\frac{1}{2}, 2}(S)$ where $u$ is the unique solution of inequality (i).

Proof. Assertion 1: $K_{1}$ is a closed, convex cone of $L_{2}(S)$. The linear continuous operator $\mathcal{A}$ defined by

$$
(v, \mathcal{A} u)=\delta(v \mid u)+(v, A u) \quad \text { for all } u, v \in X
$$

is strongly coercive on $L_{2}(S)$ since $(u, \mathcal{A} u) \geq \delta\|u\|_{2}^{2}+c\|u\|_{-\frac{1}{2}, 2}^{2}$ for all $u \in L_{2}(S)$ (cf. (2)). Thus existence and uniqueness of the solution $u^{\delta}$ of variational inequality (4) follow immediately.

Assertion 2: We set $v=0$ in (4) and get

$$
\delta\left\|u^{\delta}\right\|_{2}^{2}+\left(u^{\delta}, A u^{\delta}\right) \leq\left\|b^{\delta}\right\|_{\frac{1}{2}, 2}\left\|u^{\delta}\right\|_{-\frac{i}{2}, 2} .
$$


Thus, by (2) and Young's inequality

$$
\delta\left\|u^{\delta}\right\|_{2}^{2}+\frac{c}{2}\left\|u^{\delta}\right\|_{-\frac{1}{2}, 2}^{2} \leq c_{1}\left\|b^{\delta}\right\|_{\frac{1}{2}, 2}^{2} .
$$

This means that there exists a constant $C>0$ such that

$$
\sup _{\delta}\left\|u^{\delta}\right\|_{-\frac{1}{2}, 2} \leq C \quad \text { and } \quad \sup _{\delta} \sqrt{\delta}\left\|u^{\delta}\right\|_{2} \leq C .
$$

Assertion 3: Now, we suppose that $b^{\delta} \rightarrow b$ in $W^{\frac{1}{2}, 2}(S)$ and that $\left(\delta_{n}\right)$ is a sequence converging to zero. For simplicity we write only $\delta$ instead of $\delta_{n}$ in what follows. Then we may conclude that, at least for a subsequence, $u^{\delta}-u_{1} \in K$ in $X$ and $\sqrt{\delta} u^{\delta}-w$ in $L_{2}(S)$. By compact embedding, $\sqrt{\delta} u^{\delta} \rightarrow w$ in $X$. Since $\left(u^{\delta}\right)$ is bounded in $X$ it follows that $\sqrt{\delta} u^{\delta} \rightarrow 0$ in $X$ as $\delta \rightarrow+0$. Therefore $w=0$ and $\sqrt{\delta} u^{\delta} \rightarrow 0$ in $L_{2}(S)$.

(a) To prove $u=u_{1}$ we want to show that $u_{1}$ satisfies the inequality

$$
\left(v-u_{1}, A u_{1}\right) \geq\left(v-u_{1}, b\right) \quad \text { for all } v \in K_{1} \text {. }
$$

Then a density argument proves that $u_{1}$ is a solution of inequality (1) and the uniqueness of the solution gives $u=u_{1}$. Indeed, from (4) we get

$$
\delta\left(u^{\delta} \mid u^{\delta}\right)+\left(u^{\delta}, A u^{\delta}\right) \leq\left(u^{\delta}-v, b^{\delta}\right)+\delta\left(v \mid u^{\delta}\right)+\left(v, A u^{\delta}\right) .
$$

Since the positive bilinear form $v \mapsto(A v, v)$ is weakly sequentially lower semicontinuous (cf. Zeidler [19: Vol. 3, p. 156]) it follows from $\delta \rightarrow+0$ that

$$
\begin{aligned}
\left(u_{1}, A u_{1}\right) & \leq \liminf \left(u^{\delta}, A u^{\delta}\right) \\
& \leq \liminf \left(\left(u^{\delta}, A u^{\delta}\right)+\delta\left\|u^{\delta}\right\|^{2}\right) \\
& \leq\left(u_{1}-v, b\right)+\left(v, A u_{1}\right)
\end{aligned}
$$

for all $v \in K_{1}$. Thus (7) is proved and we have $u=u_{1}$. A well-known argument concerning subsequences (cf. Zeidler.[19: Vol. 1, p. 480]) shows that the whole sequence $\left(u^{\delta_{n}}\right)$ is weakly convergent to $u$.

(b) We prove the strong convergence $u^{\delta} \rightarrow u$ in $X$. Let' us use (8) with $v=u$ to get

$$
\begin{aligned}
(u, A u) & \leq \liminf \left(u^{\delta}, A u^{\delta}\right) \\
& \leq \lim \sup \left(u^{\delta}, A u^{\delta}\right) \\
& \leq \lim \sup \left(\left(u^{\delta}, A u^{\delta}\right)+\delta\left\|u^{\delta}\right\|^{2}\right) \\
& \leq \limsup \left(\left(u^{\delta}-u, b^{\delta}\right)+\delta\left(u \mid u^{\delta}\right)+\left(u, A u^{\delta}\right)\right) \\
& =(u, A u)
\end{aligned}
$$

and therefore $\left(\dot{u}^{\delta}, A u^{\delta}\right) \rightarrow(u, A u)$ as $\delta \rightarrow+0$. Then (2) implies

$$
c\left\|u^{\delta}-u\right\|_{-\frac{1}{2}, 2}^{2} \leq\left(u^{\delta}-u, A u^{\delta}-A u\right) \longrightarrow 0
$$

and Assertion 3 is proved 


\section{Approximation (II)}

In Section 2 we replaced the variational inequality (1) acting in $X=W^{-\frac{1}{2}, 2}(S)$ by a family of approximate variational inequalities depending on $\delta>0$ with cone $K_{1} \subseteq L_{2}(S)$ (see (4)). Now we suppose that $\delta>0$ is fixed and introduce a penalization of the negative part of the functions of $L_{2}(S)$. The aim is to get a variational inequality over the whole of $L_{2}(S)$. This variational inequality has a unique solution $u_{\varepsilon}=u_{\varepsilon}^{\delta}$ where $\varepsilon>0$ is the penalization parameter. (Since $\delta$ is fixed in this section we shall omit the supercript $\delta$.) Later, in Sections 4 and 5 we are going to derive bounds on the solutions depending neither on $\varepsilon$ nor on $\delta$ in order to get regularity results for the solution $u$ of variational inequality (1).

Suppose $\varepsilon>0$. We construct the following approximation of the variational inequality (4):

Find $u_{\varepsilon} \in L_{2}(S)$ such that

$$
\delta\left(v-u_{\varepsilon} \mid u_{\varepsilon}\right)+\left(v-u_{\varepsilon}, A u_{\varepsilon}\right)+F_{\varepsilon}(v)-F_{\varepsilon}\left(u_{\varepsilon}\right) \geq\left(v-u_{\varepsilon}, b_{\varepsilon}\right)
$$

for all $v \in L_{2}(S)$, where $b_{\varepsilon} \in W^{\frac{1}{2}, 2}(S)$ and the penalization functional $F_{\varepsilon}$ is defined by

$$
F_{\varepsilon}(v)=\frac{1}{2 \varepsilon} \int_{S}\left|v^{-}\right|^{2} d S
$$

for $v \in L_{2}(S)$, denoting for any real function $\varphi$ by $\varphi^{ \pm}$the positive and negative parts of $\varphi$, respectively, i.e. $\varphi=\varphi^{+}+\varphi^{-}$.

Parallel with (10) we consider the following variational inequality:

Find $u^{\delta} \in L_{2}(S)$ such that

$$
\delta\left(v-u^{\delta} \mid u^{\delta}\right)+\left(v-u^{\delta}, A u^{\delta}\right)+F(v)-F\left(u^{\delta}\right) \geq\left(v-u^{\delta}, b^{\delta}\right)
$$

for all $v \in L_{2}(S)$, where $F$ is the indicatrix of the convex set $K_{1}$, i.e. for $v \in L_{2}(S)$ we have $F(v)=0$ if $v \in K_{1}$ and $F(v)=+\infty$ otherwise.

We get now the following statement.

Proposition 2. Let $\delta>0$ be fixed and $b^{\delta}, b^{e} \in W^{\frac{1}{2}, 2}(S)$. Then the following assertions are true.

1. For any $\varepsilon>0$ the variational inequality (10) has exactly one solution $u_{e} \in L_{2}(S)$.

2. The variational inequality (11) has exactly one solution $u^{\delta} \in L_{2}(S)$.

3. If $M_{0}=\sup _{\varepsilon}\left\|b_{\varepsilon}\right\|_{\frac{1}{2}, 2}<+\infty$, then there exists a constant $M>0$ independent of $\delta$ such that $M=\sup _{\varepsilon}\left(\left\|u_{e}\right\|_{-\frac{1}{2}, 2}^{2}+\delta\left\|u_{e}\right\|_{2}^{2}+F_{e}\left(u_{e}\right)\right)<+\infty$.

4. $b_{e} \rightarrow b^{\delta}$ in $W^{\frac{1}{2}, 2}(S)$ as $\varepsilon \rightarrow+0$ implies $u_{e} \rightarrow u^{\delta}$ in $L_{2}(S)$ and in $W^{-\frac{1}{2}, 2}(S)$. 
Proof. Assertion 1 follows from the coercivity of the operator $\mathcal{A}$-defined by (5) and the fact that $F_{\varepsilon}(v) \geq 0$ for all $v \in L_{2}(S)$ (cf. Lions [9: Chapter 2.8.5/Theorem 8.5]). Since (11) and (4) are equivalent Assertion 2 is obvious. To prove Assertion 3 we set $v=0$ in (10). As $F_{\varepsilon}(0)=0$ we get

$$
\delta\left\|u_{\varepsilon}\right\|_{2}^{2}+\left(u_{\varepsilon}, A u_{\varepsilon}\right)+F_{\varepsilon}\left(u_{\varepsilon}\right) \leq\left\|b_{\varepsilon}\right\|_{\frac{1}{2}, 2}\left\|u_{\varepsilon}\right\|_{-\frac{1}{2}, 2}
$$

Therefore

$$
\delta\left\|u_{\varepsilon}\right\|_{2}^{2}+\frac{c}{2}\left\|u_{\varepsilon}\right\|_{-\frac{1}{2}, 2}^{2}+F_{\varepsilon}\left(u_{\varepsilon}\right) \leq c_{1}\left\|b_{\varepsilon}\right\|_{\frac{1}{2}, 2}^{2}
$$

which gives Assertion 3.

To prove Assertion 4 suppose $\varepsilon=\varepsilon_{n} \rightarrow+0$. If $\left\|b_{\varepsilon}-b^{\delta}\right\|_{\frac{1}{2} ; 2} \rightarrow 0$ we get from estimate (12) that at least for a subsequence $u_{\varepsilon} \rightarrow u_{1}$ in $L_{2}(S)$. Thus $u_{\varepsilon} \rightarrow u_{1}$ in $X$. We need to prove that $u_{1}=u^{\delta}$. From the variational inequality (10) it follows that

$$
\delta\left\|u_{\varepsilon}\right\|_{2}^{2}+\left(u_{\varepsilon}, A u_{\varepsilon}\right) \leq \delta\left(v \mid u_{\varepsilon}\right)+\left(v, A u_{\varepsilon}\right)+F_{\varepsilon}(v)-F_{\varepsilon}\left(u_{\varepsilon}\right)+\left(u_{\varepsilon}-v, b_{\varepsilon}\right)
$$

for all $v \in L_{2}(S)$. By virtue of Barbu and Precupanu [3: Theorem 2.3/p. 107] we have

$$
F_{\varepsilon}(\varphi)=\frac{1}{2 \varepsilon}\left\|\varphi-J_{\varepsilon} \varphi\right\|_{2}^{2}+F\left(J_{\varepsilon} \varphi\right)
$$

where $J_{\varepsilon}=(I+\varepsilon \partial F)^{-1}$ denotes the resolvent of $\partial F$. Then $\sup _{\varepsilon} F_{\varepsilon}\left(u_{\varepsilon}\right)<+\infty$ implies $\left\|u_{\varepsilon}-J_{\varepsilon} u_{\varepsilon}\right\| \rightarrow 0$ if $\varepsilon \rightarrow+0$. Therefore we have $J_{\varepsilon} u_{\varepsilon} \rightarrow u_{1}$ in $L_{2}(S)$ and, since the convex funtion $F$ is weakly sequentially lower semincontinuous (see [3: p. 102]),

$$
\begin{aligned}
F\left(u_{1}\right) & \leq \liminf F\left(J_{\varepsilon} u_{\varepsilon}\right) \\
& \leq \liminf \left(-\frac{1}{2 \varepsilon}\left\|u_{\varepsilon}-J_{\varepsilon} u_{\varepsilon}\right\|^{2}+F_{\varepsilon}\left(u_{\varepsilon}\right)\right) \\
& \leq \liminf F_{\varepsilon}\left(u_{\varepsilon}\right) .
\end{aligned}
$$

Since $u_{\varepsilon} \rightarrow u_{1}$ in $L_{2}(S)$ and $u_{\varepsilon} \rightarrow u_{1}$ in $\mathrm{X}$ we get from (13)

$$
\begin{aligned}
\delta\left\|u_{1}\right\|^{2} & +\left(u_{1}, A u_{1}\right) \\
& \leq \liminf \left(\delta\left\|u_{\varepsilon}\right\|_{2}^{2}+\left(u_{\varepsilon}, A u_{\varepsilon}\right)\right) \\
& \leq \lim \sup \left(\delta\left\|u_{\varepsilon}\right\|_{2}^{2}+\left(u_{\varepsilon}, A u_{\varepsilon}\right)\right) \\
& \leq \limsup \left\{\delta\left(v \mid u_{\varepsilon}\right)+\left(v, A u_{\varepsilon}\right)+F_{\varepsilon}(v)-F_{\varepsilon}\left(u_{\varepsilon}\right)+\left(u_{\varepsilon}-v, b_{\varepsilon}\right)\right\} \\
& \leq F(v)-\liminf F_{\varepsilon}\left(u_{\varepsilon}\right)+\delta\left(v \mid u_{1}\right)+\left(v, A u_{1}\right)+\left(u_{1}-v, b\right) \\
& \leq F(v)-F\left(u_{1}\right)+\delta\left(v \mid u_{1}\right)+\left(v, A u_{1}\right)+\left(u_{1}-v, b\right)
\end{aligned}
$$

for all $v \in L_{2}(S)$, i.e. $u_{1}$ is a solution of variational inequality (11). Observe that $F_{e}(v) \rightarrow F(v)$ for all $v \in L_{2}(S)$. (see Barbu and Precupanu [3: $\mathrm{p}$. 107]). Uniqueness implies $u_{1}=u^{\delta}$ 


\section{Regularity}

In this section we derive $L_{p}$-bounds for the solution $u_{\varepsilon}=u_{\varepsilon}^{\delta}$ of the variational inequality (10) that are independent of $\varepsilon$ and $\delta$. (Here again, we shall omit the supercript $\delta$.) We are going to consider $u_{\varepsilon}$ on the hyperplane part $\Gamma$ of $S$ defined in hypothesis (H2). The solution $u_{\varepsilon} \in L_{2}(S)$ satifies the inequality

$$
\delta\left(v-u_{\varepsilon} \mid u_{\varepsilon}\right)+\left(v-u_{\varepsilon}, A u_{\varepsilon}\right)+F_{\varepsilon}(v)-F_{\varepsilon}\left(u_{\varepsilon}\right) \geq\left(v-u_{\varepsilon}, b_{\varepsilon}\right)
$$

for all $v \in L_{2}(S)$. We multiply inequality (17) by the test function $v=u_{\varepsilon}+t \eta$, where $0 \neq t \in \mathbb{R}$ and $\eta \in C_{0}^{\infty}(S)$ satisfies the condition supp $\eta \subset \subset \Gamma$. Thus

$$
\delta\left(\eta \mid u_{\varepsilon}\right)+\left(\eta, A u_{\varepsilon}\right)+\frac{1}{t}\left(F_{\varepsilon}\left(u_{\varepsilon}+t \eta\right)-F_{\varepsilon}\left(u_{\varepsilon}\right)\right)\left\{\begin{array}{l}
\geq \\
\leq
\end{array}\right\}\left(\eta, b_{\varepsilon}\right) \text { for } t\left\{\begin{array}{l}
> \\
<
\end{array}\right\} 0
$$

From

$$
\lim _{t \rightarrow 0} \frac{1}{t}\left(F_{\varepsilon}\left(u_{\varepsilon}+t \eta\right)-F_{\varepsilon}\left(u_{\varepsilon}\right)\right)=\varepsilon^{-1} \int_{\Gamma} \eta u_{\varepsilon}^{-} d S
$$

it follows that

$$
\delta \int_{S} \eta u_{\varepsilon} d S+\int_{S} \eta A u_{\varepsilon} d S+\varepsilon^{-1} \int_{S} \eta u_{\varepsilon}^{-} d S=\int_{S} \eta b_{\varepsilon} d S
$$

for all $\eta \in C_{0}^{\infty}(S)$ and by approximation for all $\eta \in L_{2}(S)$ with supp $\eta \subset \subset \Gamma$. Since $\eta$ can be chosen arbitrarily we get

$$
\delta u_{\varepsilon}+A u_{\varepsilon}+\varepsilon^{-1} u_{\varepsilon}^{-}=b_{\epsilon} \quad \text { in } L_{2}^{\text {loc }}(\Gamma) .
$$

4.1 (Localization and preliminary regularity). In the following we are going to use local properties of pseudodifferential operators. We choose an open subset $U \subset \subset \Gamma$ and an arbitrary but fixed test function $\varphi \in C_{0}^{\infty}(U)$ with $\varphi \geq 0$. Setting $g_{c}=\varphi u_{\varepsilon}$, relation (19) gives

$$
\delta g_{\varepsilon}+\varphi A u_{\varepsilon}+\varepsilon^{-1} g_{\varepsilon}^{-}=\varphi b_{e}=: \widetilde{b}_{\varepsilon} .
$$

Remark that supp $\tilde{b}_{e} \subset \dot{U}$. Furthermore we choose a function $\mu \in C_{0}^{\infty}(U)$ such that $\mu \equiv 1$ on an open set $W \subset \subset U$ with $K_{\varphi}=\operatorname{supp} \varphi \subset W$. Then relation (20) may be written in the form

$$
\delta g_{\varepsilon}+(\varphi A \mu) u_{\varepsilon}+\varepsilon^{-1} g_{\varepsilon}^{-}=\tilde{b}_{\varepsilon}-\varphi A(1-\mu) u_{\varepsilon}=\tilde{b}_{\varepsilon}+R_{1} u_{\varepsilon}=\tilde{b}_{\varepsilon}+\mu R_{1} u_{\varepsilon}
$$

where $R_{1}=-\varphi A(1-\mu)$ is a so-called regularizing $\psi$ do: $R_{1} \in \Psi^{-\infty}(S)$ (see Dieudonné [4: Vol. 7, Prop. 23.26.11/p. 212]). Therefore $R_{1}: W^{-\frac{1}{2}, 2}(S) \longrightarrow W^{m, 2}(U) \subset W^{m, 2}(S)$ is a continuous operator for all $m \in \mathbb{N}$.

Next we make use of the principal symbol $\sigma_{-1}(A)$ defined in hypothesis (H2). Let us agree to write $x \in \mathbb{R}^{N}$ and $\xi \in \mathbb{R}^{N}$ in the following instead of $x^{\prime}$ and $\xi^{\prime}$, respectively. Since the principal symbols of both $\varphi A \mu$ and $\mu A \varphi$ are the same: $\sigma_{-1}(\varphi A \mu)(x, \xi) \doteq$ $\sigma_{-1}(\mu A \varphi)(x, \xi)=\varphi(x)|\xi|^{-1}$, we only get a perturbation of order -2 exchanging $\varphi$ and 
$\mu$ in the term $(\varphi A \mu)$ of (21): $\varphi A \mu=\mu A \varphi+P_{-2}$ where $P_{-2} \in \Psi^{-2}(U)$ is a proper $\psi$ do of order -2 . Thus

$$
\delta g_{\varepsilon}+(\mu A \varphi) u_{\varepsilon}+\varepsilon^{-1} g_{\varepsilon}^{-}=\tilde{b}_{\varepsilon}+\mu R_{1} u_{\varepsilon}+P_{-2} g_{e}=: f_{\epsilon}
$$

By the mapping properties of proper $\psi$ do's, we see that $P_{-2}: W^{-\frac{1}{2}, 2}(U) \longrightarrow W^{\frac{3}{2}, 2}(U)$ is a continuous linear mapping. Introducing a third cut-off function $\mu_{1}$ such that $\mu_{1} \equiv 1$ on $\operatorname{supp} \mu$ we can re-write (22) as

$$
\delta g_{\epsilon}+\left(\mu A \mu_{1}\right) g_{\varepsilon}+\varepsilon^{-1} g_{\varepsilon}^{-}=f_{\varepsilon} .
$$

The principal symbol of $\mu A \mu_{1}$ on $\Gamma$ is $\sigma_{-1}\left(\mu A \mu_{1}\right)=\mu(x)|\xi|^{-1}$.

Let us fix $\varepsilon>0$ and study the individual function $g_{e}$ for a moment.

Lemma 1. Let us assume $b_{\varepsilon} \in W_{\text {loc }}^{1, p}(\Gamma)$ for all $p<+\infty$. Then $g_{\varepsilon}=\varphi u_{\varepsilon}^{\delta} \in W^{1, p}(U)$ for all $\varepsilon, \delta>0$ and $p<+\infty$.

Proof. The solution $u_{\varepsilon}$ of inequality (17) belongs to $L_{2}(S)$. Therefore $f_{\varepsilon} \in W^{1,2}(U)$. From Treves [15: Theorem 2.1/p. 16] we get $\left(\mu A \mu_{1}\right) g_{\epsilon} \in W^{1,2}(U)$ and relation (23) gives the inclusion

$$
\delta g_{\epsilon}+\varepsilon^{-1} g_{e}^{-} \in W^{1,2}(U)
$$

Therefore $\delta g_{\varepsilon}^{+}$and $\left(\delta+\varepsilon^{-1}\right) g_{\varepsilon}^{-}$both belong to $W^{1,2}(U)$, and $g_{\varepsilon} \in W^{1,2}(U)$ for each fixed pair $\delta, \varepsilon>0$. From the embedding theorem it follows that $g_{\varepsilon} \in L_{p_{1}}(U)$ with $p_{1}=\frac{2 N}{N-2}$ for $N \geq 3$ and $p_{1}<+\infty$ arbitrary for $N=2$. From the same argument we derive the inclusion $f_{e},\left(\mu A \mu_{1}\right) g_{\varepsilon} \in W^{1, p_{1}}(U)$ and finally $g_{\varepsilon} \in W^{1, p_{1}}(S) \subset L_{p_{2}}(U)$ with $p_{2}=\frac{2 N}{N-4}$ for $N \geq 5$ and $p_{2}<+\infty$ arbitrary for $N \leq 4$. Repeating the argument we conclude that for each $\varepsilon, \delta>0$

$$
g_{\varepsilon}=\varphi u_{\varepsilon}^{\delta} \in W^{1, p}(U) \quad \text { for all } p<+\infty
$$

Then it follows from the embedding theorem that $g_{e} \in C^{\beta}(U)$ for all $\beta \in(0,1)$

4.2 ( $\mathrm{L}_{p}$-regularity). We intend first to apply a $\psi$ do $P$ with principal symbol $|\xi|$ to equality (23). Then we multiply it by the test function $\left\langle g_{\varepsilon}\right\rangle^{p-1}=\left|\dot{g}_{\varepsilon}\right|^{p-2} g_{\varepsilon}$. In order to avoid additional regularizing terms containing $\varepsilon^{-1} g_{e}^{-}$we need some preparation. For this define

$$
(P v)(x)=\int_{\mathbb{R}^{N}} e^{i x \xi} \chi(\xi)|\xi| \hat{v}(\xi) \frac{d \xi}{(2 \pi)^{N}}
$$

for $v \in C_{0}^{\infty}\left(\mathbb{R}^{N}\right)$, where $\chi \in C^{\infty}\left(\mathbb{R}^{N}\right)$ is a cut-off function characterized, e.g., by

$$
\chi(\xi)= \begin{cases}0 & \text { if }|\xi|<1 \\ 1 & \text { if }|\xi| \geq 2\end{cases}
$$

Now we put $\int P v \cdot w d x$ into a form adapted for considerations of the positive and negative part of the functions involved. Taking real functions $v, w \in C_{0}^{\infty}\left(\mathbb{R}^{N}\right)$ the 
theorem of Fubini gives

$$
\begin{aligned}
(P v, w) & =\int_{\mathbb{R}^{N}}\left(\int_{\mathbb{R}^{N}} e^{i x \xi} \chi(\xi)|\xi| \hat{v}(\xi) \frac{d \xi}{(2 \pi)^{N}}\right) w(x) d x \\
& =\int_{\mathbb{R}^{N}} \chi(\xi)|\xi| \hat{v}(\xi) \overline{\hat{w}(\xi)} \frac{d \xi}{(2 \pi)^{N}} \\
& =\int_{\mathbb{R}^{N}}|\xi| \hat{v}(\xi) \overline{\hat{w}(\xi)} \frac{d \xi}{(2 \pi)^{N}}+\int_{\mathbb{R}^{N}}(\chi(\xi)-1)|\xi| \hat{v}(\xi) \overline{\hat{w}(\xi)} \frac{d \xi}{(2 \pi)^{N}} \\
& =: I_{1}+I_{2} .
\end{aligned}
$$

The operator $R_{2}$ defined by

$$
\left(R_{2} v\right)(x)=\int_{\mathbb{R}^{N}} e^{i x \xi}(\chi(\xi)-1)|\xi| \hat{v}(\xi) \frac{d \xi}{(2 \pi)^{N}} \quad \text { for } \quad v \in C_{0}^{\infty}\left(\mathbb{R}^{N}\right)
$$

is regularizing: $R_{2} \in \Psi^{-\infty}\left(\mathbb{R}^{N}\right)$, since the amplitude $\chi(\xi)-1$ vanishes outside the ball $B_{2}(0)$ (cf. Dieudonné [4: Remark 23.19.5(iii)/p.149]). Applying Parseval's equality to $I_{1}$ we get

$$
I_{1}=a \iint_{\mathbb{R}^{N} \times \mathbb{R}^{N}}|x-y|^{-N-1}(v(x)-v(y))(w(x)-w(y)) d x d y
$$

where $a=a(N)>0$ is a constant (see Wloka [18: p. 97] and Hörmander [5: Vol. 1/p. 241]). We stress that both integrals $I_{1}$ and $I_{2}$ depend on $v$ and $w$. We have $\left(R_{2} v, w\right)=I_{2}$ and define an operator $J_{1}$ by

$$
\left(J_{1} v, w\right)=I_{1}=a \iint_{\mathbb{R}^{N} \times \mathbb{R}^{N}}|x-y|^{-N-1}(v(x)-v(y))(w(x)-w(y)) d x d y
$$

for all $v, w \in C_{0}^{\infty}\left(\mathbb{R}^{N}\right)$ to get

$$
\left(J_{1} v, w\right)=(P v, w)-\left(R_{2} v, w\right)
$$

We now prove $L_{p}$-regularity of the solution $u$ of the variational inequality (1).

Theorem 1. Let $2 \leq p<+\infty$ and $b \in W^{\frac{1}{2}, 2}(S) \cap W_{l o c}^{1, p}(\Gamma)$. Then $u \in L_{\mathrm{p}}^{\text {loc }}(\Gamma)$.

Remark 1. For $2 \leq p<+\infty$, the inclusion $b \in W^{1, p}(S)$ implies the inclusion $u \in L_{p}(S)$ if after a coordinate transform the operator $A$ has the principal symbol (3) in each coordinate patch of a partition of unity on $S$.

Proof of Theorem 1. To prove the theorem we consider the approximate problems and derive uniform bounds for the solutions $u_{e}=u_{\varepsilon}^{\delta}$ of inequality. (10) and $u^{\delta}$ of inequality (4).

(a) For simplicity we set $b^{\delta}=b \in W^{\frac{1}{2}, 2}(S) \cap W_{\text {loc }}^{1, p}(\Gamma)$. By approximation, we may assume that the family $\left(b_{e}\right)$ belongs to $W^{\frac{1}{2}, 2}(S) \cap W_{l o c}^{1, q}(\Gamma)$ for all $q<+\infty$ and, 
furthermore, $b_{\varepsilon} \rightarrow b^{\delta}$ in $W^{\frac{1}{2}, 2}(S)$ and in $W_{\text {loc }}^{1, p}(\Gamma)$ as $\varepsilon \rightarrow+0$. In particular, for äny open set $O \subset \subset \Gamma$

$$
\sup _{\varepsilon^{\prime}}\left\|b_{\varepsilon}\right\|_{1, p, O} \leq M=M(O)<+\infty \text {. }
$$

It follows from Lemma 1 that $g_{\varepsilon} \in W^{1, q}(U)$ for all $q<+\infty$. Therefore also $g_{\varepsilon}^{-} \epsilon$ $W^{1, q}(U)$ for all $q<+\infty$. Suppose $q>N$, arbitrary. Then $W^{1, q}(U)$ is a Banach algebra (see Adams [1: p. 115]) and it follows that $\left\langle g_{\varepsilon}\right\rangle^{p-1}=\left|g_{\varepsilon}\right|^{p-2} g_{\varepsilon} \in W^{1, q}(U)$ for each $q \geq 2$. It is our goal to show that (29) implies

$$
\sup _{\varepsilon}\left\|g_{\varepsilon}\right\|_{p} \leq M_{1}<+\infty
$$

where the constant $M_{1}$ is independent of $\delta$. This gives the local boundedness of $u_{\varepsilon} \epsilon$ $L_{p}(\Gamma)$. In fact, we may choose $\varphi$ such that $\varphi \equiv 1$ on any open set $V \subset \subset U$ and estimation (30) implies

$$
\sup _{e}\left\|u_{e}\right\|_{p, V} \leq M_{1}<+\infty \text {. }
$$

(b) We apply operator $J_{1}$ to equality (23) and multiply it by $h_{\varepsilon}=\left\langle g_{\varepsilon}\right\rangle^{p-1}$ to get

$$
\delta\left(J_{1} g_{\varepsilon}, h_{\varepsilon}\right)+\left(J_{1}\left(\mu A \mu_{1}\right) g_{\varepsilon}, h_{\varepsilon}\right)+\varepsilon^{-1}\left(J_{1} g_{\varepsilon}^{-}, h_{\varepsilon}\right)=\left(J_{1} f_{\varepsilon}, h_{\varepsilon}\right)
$$

that is

$$
\begin{aligned}
L_{1}+L_{2}+L_{3}:= & \delta a \iint|x-y|^{-N-1}\left(g_{\varepsilon}(x)-g_{\varepsilon}(y)\right)\left(h_{\varepsilon}(x)-h_{\varepsilon}(y)\right) d x d y \\
& +\left(P\left(\mu A \mu_{1}\right) g_{\varepsilon}, h_{\varepsilon}\right) \\
& +\varepsilon^{-1} a \iint|x-y|^{-N-1}\left(g_{\varepsilon}^{-}(x)-g_{\varepsilon}^{-}(y)\right)\left(h_{\varepsilon}(x)-h_{\varepsilon}(y)\right) d x d y \\
= & \left(\left(P-R_{2}\right) f_{\varepsilon}, h_{\varepsilon}\right)+\left(R_{2}\left(\mu A \mu_{1}\right) g_{\varepsilon}, h_{\varepsilon}\right) .
\end{aligned}
$$

Now we have to consider the terms $L_{1}, L_{2}$ and $L_{3}$ of (32) separately. The function $t \mapsto|t|^{p-2} t$ is uniformly monotone for $p \geq 2$ :

$$
\left(|s|^{p-2} s-|t|^{p-2} t\right)(s-t) \geq c|s-t|^{p} \quad \text { for all } s, t \in \mathbb{R}
$$

where $c>0$ is a constant (cf. Zeidler [19: Vol. 2/p. 503]). Then

$$
\begin{aligned}
& L_{1}=\delta a \iint|x-y|^{-N-1}\left(g_{\varepsilon}(x)-g_{\varepsilon}(y)\right)\left(\left|g_{\varepsilon}(x)\right|^{p-2} g_{\varepsilon}(x)-\left|g_{\varepsilon}(y)\right|^{p-2} g_{\varepsilon}(y)\right) d x d y \\
& \quad \geq \delta c a \iint|x-y|^{-N-1}\left|g_{\varepsilon}(x)-g_{\varepsilon}(y)\right|^{p} d x d y \\
& \quad=\delta c a\left|g_{\varepsilon}\right|_{\frac{1}{p}, p}^{p} \quad \cdots \quad
\end{aligned}
$$

The third term $L_{3}$ in (32) is the penalization term. Oberserving that

$$
\left(|s|^{p-2} s-|t|^{p-2} t\right)\left(s^{-}-t^{-}\right) \geq\left(\left|s^{-}\right|^{p-2} s^{-}-\left|t^{-}\right|^{p-2} t^{-}\right)\left(s^{-}-t^{-}\right)
$$


it follows from (33) that

$$
\begin{aligned}
L_{3} & =\dot{\varepsilon}^{-1} a \iint|x-y|^{-N-1}\left(g_{\varepsilon}^{-}(x)-g_{\varepsilon}^{-}(y)\right)\left(h_{e}(x)-h_{\varepsilon}(y)\right) d x d y \\
& \geq \varepsilon^{-1} c a \iint|x-y|^{-N-1}\left|g_{\varepsilon}^{-}(x)-g_{\varepsilon}^{-}(y)\right|^{p} d x d y \\
& =\varepsilon^{-1} c a\left|g_{\varepsilon}^{-}\right|_{\frac{1}{p}, p}^{p}
\end{aligned}
$$

The second term of $L_{2}=\left(P\left(\mu A \mu_{1}\right) g_{\varepsilon}, h_{\varepsilon}\right)$ of (32) contains the composition of $P \in$ $\Psi^{1}(U)$ and the proper $\psi$ do $\mu A \mu_{1} \in \Psi^{-1}(U)$. The principal symbol of $P\left(\mu A \mu_{1}\right) \in \Psi^{0}(U)$ is $\sigma_{0}\left(P\left(\mu A \mu_{1}\right)\right)(x, \xi)=\chi(\xi) \mu(x)$. Thus there exisț a $\psi$ do $P_{-1} \in \Psi^{-1}(U)$ such that

$$
\begin{aligned}
& \int\left(P \mu A \mu_{1}\right)(v) \cdot w d x
\end{aligned}
$$

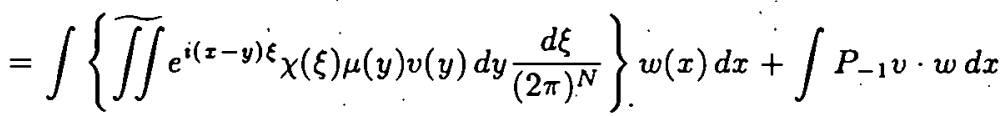

$$
\begin{aligned}
& =\int\left(\int e^{i x \xi} \chi(\xi) \hat{v}(\xi) \frac{d \xi}{(2 \pi)^{N}}\right) w(x) d x+\left(P_{-1} v, w\right) . \\
& =\int\left(\int e^{i x \xi} \hat{v}(\xi) \frac{d \xi}{(2 \pi)^{N}}\right) w(x) d x \\
& +\int\left(\int_{\mathbb{R}^{N}} e^{i x \xi}(\chi(\xi)-1) \hat{v}(\xi) \frac{d \xi}{(2 \pi)^{N}}\right) w(x) d x+\left(P_{-1} v, w\right) \\
& =\int v w d x+\left(R_{3} v, w\right)+\left(P_{-1} v, w\right)
\end{aligned}
$$

for all $v, w \in C_{0}^{\infty}(W)$ where $\widetilde{\iint}$ denotes an oscillatory integral and $R_{3}$ is regularizing by the argument already used for $R_{2}$. Then, by approximation,

$$
L_{2}=\int_{\Gamma}\left|g_{\varepsilon}\right|^{p} d x+\left(R_{3} g_{\varepsilon} ; h_{\varepsilon}\right)+\left(P_{-1} g_{\varepsilon}, h_{\varepsilon}\right) .
$$

By Hölder's inequality, equations (32) and (34) together give

$$
\begin{aligned}
\delta c a\left|g_{e}\right|_{\frac{1}{p}, p}^{p}+\left\|g_{e}\right\|_{p}^{p}+\varepsilon^{-1} c a\left|g_{e}^{-}\right|_{\frac{1}{p}, p}^{p} & \\
\leq & \left(\left\|\left(P-R_{2}\right) f_{\varepsilon}\right\|_{p}+\left\|R_{2}\left(\mu A \mu_{1}\right) g_{e}\right\|_{p}+\left\|R_{3} g_{\varepsilon}\right\|_{p^{\prime}}+\left\|P_{-1} g_{e}\right\|_{p}\right)\left\|g_{e}\right\|_{p}^{p-1} \\
\leq & C\left(\left\|\varphi b_{\varepsilon}\right\|_{1, p, W}+\left\|\left(P-R_{2}\right) R_{1} u_{\varepsilon}\right\|_{p, W}+\left\|P_{-2} g_{\varepsilon}\right\|_{1, p, W}\right. \\
& \left.+\left\|R_{2}\left(\mu A \mu_{1}\right) g_{e}\right\|_{p, W}+\left\|R_{3} g_{e}\right\|_{p, W}+\left\|P_{-1} g_{e}\right\|_{p, W}\right)\left\|g_{e}\right\|_{p}^{p-1}
\end{aligned}
$$

since $K_{\varphi}=\operatorname{supp} \varphi \subset W \subset \subset U$. Young's inequality and Proposition 2 imply

$$
\begin{aligned}
\delta\left|g_{e}\right|_{\frac{1}{p}, p}^{p} & +\left\|g_{\varepsilon}\right\|_{p}^{p}+\varepsilon^{-1}\left|g_{e}^{-}\right|_{\frac{1}{p}, p}^{p} \\
& \leq C\left(\left\|b_{\varepsilon}\right\|_{1, p, W}^{p}+\left\|u_{\varepsilon}\right\|_{-\frac{1}{2}, 2, S}^{p}+\left\|P_{-2} g_{\varepsilon}\right\|_{1, p, W}^{p}+\left\|g_{\varepsilon}\right\|_{-\frac{1}{2}, 2}^{p}+\left\|P_{-1} g_{e}\right\|_{p, W}^{p}\right) \\
& \leq C\left(1+\left\|P_{-2} g_{e}\right\|_{1, p, W}^{p}+\left\|P_{-1} g_{e}\right\|_{p, W}\right)
\end{aligned}
$$


since $R_{1}$ and $R_{2}$ are regularizing.

(c) We are:going to apply a bootstrap argument. Using the embedding theorem and the fact that $P_{-1}: W_{\text {comp }}^{-\frac{1}{2}, 2}(U) \longrightarrow W_{l o c}^{\frac{1}{2}, 2}(U)$ and $P_{-2}: W_{\text {comp }}^{-\frac{1}{2}, 2}(U) \longrightarrow W_{\text {loc }}^{\frac{3}{2}, 2}(U)$ are continuous linear mappings we get

$$
\begin{gathered}
\left\|P_{-1} g_{\varepsilon}\right\|_{q_{1}, W} \leq c_{1}\left\|P_{-1} g_{e}\right\|_{\frac{1}{2}, 2, W} \leq c_{2}\left\|g_{e}\right\|_{-\frac{1}{2}, 2} \\
\left\|P_{-2} g_{e}\right\|_{1, q_{1}, W} \leq c_{1}\left\|P_{-2} g_{e}\right\|_{\frac{3}{2}, 2, W} \leq c_{2}\left\|g_{e}\right\|_{-\frac{1}{2}, 2}
\end{gathered}
$$

for some constants $c_{1}>0$ and $c_{2}>0$, where $q_{1}=\frac{2 N}{N-1}$. We stress that these constants depend upon $W$ and $K_{\varphi}$, but neither on $\varepsilon$ nor on $\delta$. It follows from (35) with $p=q_{1}$ that

$$
\sup _{e}\left(\delta\left|g_{e}\right|_{\frac{1}{q_{1}}, q_{1}}^{q_{1}}+\left\|g_{e}\right\|_{q_{1}}^{q_{1}}+\varepsilon^{-1}\left|g_{e}^{-}\right|_{\frac{1}{q_{1}}, q_{1}}^{q_{1}}\right)<+\infty \text {. }
$$

This implies $\sup _{\varepsilon}\left\|g_{\varepsilon}\right\|_{q_{1}}<+\infty$. As in the first step we get

$$
\sup _{e}\left\{\left\|P_{-2} g_{e}\right\|_{2, g_{1}, W}+\left\|P_{-1} g_{e}\right\|_{1, g_{1}, W}\right\}<+\infty .
$$

With $q_{2}=\frac{2 N}{N-3}$ the embedding theorem implies

$$
\left\|P_{-2} g_{\varepsilon}\right\|_{1, q_{2}, W} \leq c_{3}\left\|P_{-2} g_{\varepsilon}\right\|_{2, q_{1}, U} \quad \text { and } \quad\left\|P_{-1} g_{\varepsilon}\right\|_{q_{2}, W} \leq c_{3}\left\|P_{-1} g_{\varepsilon}\right\|_{1, q_{1}, W}
$$

and we get from (35) with $p=q_{2}$

$$
\sup _{\varepsilon, \delta}\left(\delta\left|g_{\varepsilon}\right|_{\frac{1}{q_{2}}, q_{2}}^{q_{2}}+\left\|g_{\varepsilon}\right\|_{q_{2}}^{q_{2}}+\varepsilon^{-1}\left|g_{\varepsilon}^{-}\right|_{\frac{1}{q_{2}}, q_{2}}^{q_{2}}\right)<+\infty
$$

We can repeat this procedure as far as $q_{j} \leq p$. In the last step we get

$$
\sup _{\varepsilon}\left(\delta\left|g_{e}\right|_{\frac{1}{p}, p}^{p}+\left\|g_{e}\right\|_{p}^{p}+\varepsilon^{-1}\left|g_{e}^{-}\right|_{\frac{1}{p}, p}^{p}\right) \leq M_{1}<+\infty
$$

where the estimates used above show that the constant $M_{1}$ is independent of $\delta>0$. This proves estimations (30) and (31).

(d) Let $\varepsilon_{n} \rightarrow+0$ for fixed $\delta>0$. Since $\sup _{n}\left\|g_{e_{n}}\right\|_{p} \leq M_{1}$ we can extract a subsequence with $\varphi u_{\varepsilon} \rightarrow g^{\delta}$ in $L_{p}(U)$. As $u_{\varepsilon} \rightarrow u^{\delta}$ in $L_{2}(S)$ (Proposition 2) we conclude that $g^{\delta}=\varphi u^{\delta} \in L_{p}(S)$, i.e. $u^{\delta} \in L_{p}^{\text {loc }}(\Gamma)$. Let $\varphi \equiv 1$ on $V$. The weak sequential lower semicontinuity of the norm gives $\left\|u^{\delta}\right\|_{p, V} \leq\left\|\varphi u^{\delta}\right\|_{p} \leq M_{1}$ for $V \subset \subset U$.

(e) If $\delta_{n} \rightarrow+0$, there exists a subsequence such that $\varphi u^{\delta} \rightarrow u_{0}$ in $L_{p}(S)$ and $\varphi u^{\delta} \rightarrow u_{0}$ in $W^{-\frac{1}{2}, 2}(U)$. Proposition 1 gives $\varphi u^{\delta} \rightarrow \varphi u$ in $W^{-\frac{1}{2}, 2}(S)$. Consequently $u_{0}=\varphi u \in L_{p}(U)$, and it follows that $u \in L_{p}^{\text {loc }}(\Gamma)$ with $\|u\|_{p}, V \leq\|\varphi u\|_{p} \leq M_{1}$ for $V \subset \subset U$ 


\section{5. $\mathrm{L}_{\infty}$-regularity}

5.1. To prove $L_{\infty}$-regularity for the solutions $u_{\varepsilon}$ of equation (19) we apply a method from the classical theory of differential equations due to Stampacchia. It depends on estimates for the size of level sets. As in Subsection 4.2 we begin with a kind of differentiation of equation (23). Here we are going to use the operator

$$
\left(P^{\gamma} v\right)(x):=\int_{\mathbb{R}^{N}} e^{i z \xi} \chi(\xi)|\xi|^{\gamma} \hat{v}(\xi) \frac{d \xi}{(2 \pi)^{N}}
$$

for $v \in C_{0}^{\infty}\left(\mathbb{R}^{N}\right)$ where $1<\gamma<2$ and $\chi \in C^{\infty}\left(\mathbb{R}^{N}\right)$ is the same function as in Subsection 4.2. For $g_{\varepsilon}=\varphi u_{\varepsilon}$ we have the following estimate.

Lemma 2. Suppose $b_{\varepsilon} \in W^{\gamma, 2}(U)$ for some $\gamma \in(1,2)$. Then there exist appropriate $\psi$ do's $Q_{\gamma}$ and $Q_{\gamma-2}$ from $\Psi^{\gamma}(U)$ and $\Psi^{\gamma-2}(U)$, repectively, such that

$$
\begin{aligned}
\delta a|| g_{e}(x) & -k]\left.^{+}\right|_{\frac{\gamma}{2}, 2} ^{2}+a\left|\left[g_{e}(x)-k\right]^{+}\right|_{\frac{\gamma-1}{2}, 2}^{2} \\
& \leq \int_{U}\left(\left|Q_{\gamma} f_{\varepsilon}\right|+\left|Q_{\gamma-2} g_{e}\right|\right)\left[g_{e}(x)-k\right]^{+} d x .
\end{aligned}
$$

Proof. (a) For $v, w \in C_{0}^{\infty}\left(\mathbb{R}^{N}\right)$ we get

$$
\begin{aligned}
\left(P^{\gamma} v, w\right) & =\int_{\mathbb{R}^{N}}\left(\int_{\mathbb{R}^{N}} e^{i x \xi} \chi(\xi)|\xi|^{\gamma} \hat{v}(\xi) \frac{d \xi}{(2 \pi)^{N}}\right) w(x) d x \\
& =\int_{\mathbb{R}^{N}}|\xi|^{\gamma} \hat{v}(\xi) \overline{\hat{w}(\xi)} \frac{d \xi}{(2 \pi)^{N}}+\int_{\mathbb{R}^{N}}(\chi(\xi)-1)|\xi|^{\gamma} \hat{v}(\xi) \overline{\hat{w}(\xi)} \frac{d \xi}{(2 \pi)^{N}} \\
& =: I_{1}^{\gamma}+I_{2}^{\gamma} .
\end{aligned}
$$

Concerning the integral $I_{2}^{\gamma}$ we observe that the operator $R_{2}^{\gamma}$ defined by

$$
\left(R_{2}^{\gamma} v\right)(x)=\int_{\mathbf{R}^{N}} e^{i x \xi}(\chi(\xi)-1)|\xi|^{\gamma} \hat{v}(\xi) \frac{d \xi}{(2 \pi)^{N}}
$$

for $v \in C_{0}^{\infty}\left(\mathbb{R}^{N}\right)$ is regularizing, whereas Parseval's inequality implies

$$
I_{1}^{\gamma}=a \iint_{\mathbb{R}^{N} \times \mathbb{R}^{N}}|x-y|^{-N-\gamma}(v(x)-v(y))(w(x)-w(y)) d x d y
$$

with $a=a(\gamma, N)>0$. Defining

$$
\begin{aligned}
\left(J_{\gamma} v, w\right) & =I_{1}^{\gamma} \\
& =a \iint_{\mathbb{R}^{N} \times \mathbb{R}^{N}}|x-y|^{-N-\gamma}(v(x)-v(y))(w(x)-w(y)) d x d y
\end{aligned}
$$

for all $v, w \in C_{0}^{\infty}\left(\mathbb{R}^{N}\right)$ we get

$$
\left(J_{\gamma} v, w\right)=\left(P^{\gamma} v, w\right)-\left(R_{2}^{\gamma} v, w\right)
$$


(b) The application of the operator $J_{\gamma}$ to equality (23) and scalar multiplication by a test function $h_{\epsilon}$ gives

$$
\begin{aligned}
L_{1}+L_{2}+L_{3} & :=\delta a \iint|x-y|^{-N-\gamma}\left(g_{\varepsilon}(x)-g_{\varepsilon}(y)\right)\left(h_{\varepsilon}(x)-h_{\varepsilon}(y)\right) d x d y \\
& +\left(P^{\gamma}\left(\mu A \mu_{1}\right) g_{\varepsilon}, h_{\varepsilon}\right) \\
& +\varepsilon^{-1} a \iint|x-y|^{-N-\gamma}\left(g_{\varepsilon}^{-}(x)-g_{\varepsilon}^{-}(y)\right)\left(h_{\varepsilon}(x)-h_{\varepsilon}(y)\right) d x d y \\
& =\left(\left(P^{\gamma}-R_{2}^{\gamma}\right) f_{\varepsilon}, h_{\varepsilon}\right)+\left(R_{2}^{\gamma}\left(\mu A \mu_{1}\right) g_{\varepsilon}, h_{\varepsilon}\right) .
\end{aligned}
$$

For $k \geq 0$, choose $h_{\varepsilon}=\left[g_{\varepsilon}-k\right]^{+} \in W^{\frac{\gamma}{2}, 2}(U)$ in (45). It follows that supp $\left[g_{\varepsilon}(x)-k\right]^{+} \subseteq$ $\operatorname{supp} \varphi$ for $k \geq 0$. We first get

$$
\begin{aligned}
L_{1}= & \delta a \iint|x-y|^{-N-\gamma}\left(\left[g_{\varepsilon}(x)-k\right]-\left[g_{\varepsilon}(y)-k\right]\right) \\
& \times\left(\left[g_{\varepsilon}(x)-k\right]^{+}-\left[g_{\varepsilon}(y)-k\right]^{+}\right) d x d y \\
\geq & \delta a \iint|x-y|^{-N-\gamma}\left|\left[g_{\varepsilon}(x)-k\right]^{+}-\left[g_{\varepsilon}(y)-k\right]^{+}\right|^{2} d x d y \\
= & \delta a\left|\left[g_{c}-k\right]^{+}\right|_{\frac{\gamma}{2}, 2}^{2} .
\end{aligned}
$$

Observing that

$$
\left(s^{-}-t^{-}\right)\left([s-k]^{+}-[t-k]^{+}\right) \geq 0 \quad \text { for all } s, t \in \mathbb{R}
$$

we see that

$$
\begin{aligned}
L_{3}= & \varepsilon^{-1} a \iint|x-y|^{-N-\gamma}\left(g_{\varepsilon}^{-}(x)-g_{\varepsilon}^{-}(y)\right) \\
& \left.\times\left(\mid g_{\varepsilon}(x)-k\right]^{+}-\left[g_{\varepsilon}(y)-k\right]^{+}\right) d x d y \\
\geq & 0 .
\end{aligned}
$$

In the second term $L_{2}$ of (45), the principal symbol of the composition $P^{\gamma}\left(\mu A \mu_{1}\right) \in$ $\Psi^{\gamma-1}(U)$ is $\sigma_{\gamma-1}\left(P^{\gamma}\left(\mu A \mu_{1}\right)\right)(x, \xi)=\mu(x)|\xi|^{\dot{\gamma}-1} \chi(\xi)$. It follows that there exists a $\psi$ do $P_{\gamma-2} \in \Psi^{\gamma-2}(U)$ such that $P^{\gamma}\left(\mu A \mu_{1}\right)=P^{\gamma-1} \mu+P_{\gamma-2}$ where $P^{\gamma-1} \in \Psi^{\gamma-1}(U)$ is defined by (40) with $\gamma$ replaced by $\gamma-1$. Thus (44) with $\gamma-1$ instead of $\gamma$ gives

$$
\begin{aligned}
L_{2}= & \left(P^{\gamma-1} g_{\varepsilon}, h_{\varepsilon}\right)+\left(P_{\gamma-2} g_{\varepsilon}, h_{\varepsilon}\right) \\
= & a \iint|x-y|^{-N-\gamma+1}\left(\left[g_{\varepsilon}(x)-k\right]-\left[g_{\varepsilon}(y)-k\right]\right)\left(\left[g_{\varepsilon}(x)-k\right]^{+}-\left[g_{\varepsilon}(y)-k\right]^{+}\right) \\
& +\left(R_{3} g_{\varepsilon}, h_{\varepsilon}\right)+\left(P_{\gamma-2} g_{\varepsilon}, h_{\varepsilon}\right) \\
\geq & a\left|\left[g_{\varepsilon}-k\right]^{+}\right|_{\frac{\gamma-1}{2}, 2}+\left(R_{3} g_{\varepsilon}, h_{\varepsilon}\right)+\left(P_{\gamma-2} g_{\varepsilon}, h_{\varepsilon}\right) .
\end{aligned}
$$

The regularizing operator $R_{3}=R_{2}^{\gamma-1}$ arises from (44). Observe that $\mu \equiv 1$ on $K_{\varphi}=$ $\operatorname{supp} \varphi$. Summarizing we get

$$
\begin{aligned}
\delta a\left|\left[g_{\varepsilon}-k\right]^{+}\right|_{\frac{\gamma}{2}, 2}^{2}+a|| g_{\varepsilon}-\left.\left.k\right|^{+}\right|_{\frac{\gamma-1}{2}, 2} ^{2} \\
\leq \int_{U}\left\{\left(\left(P^{\gamma}-R_{2}^{\gamma}\right) f_{\varepsilon}\right)+\left(R_{2}^{\gamma}\left(\mu A \mu_{1}\right) g_{\varepsilon}-P_{\gamma-2} g_{\varepsilon}-R_{3} g_{\varepsilon}\right)\right\}\left[g_{\varepsilon}(x)-k\right]^{+} d x \\
\quad=\int_{U}\left(Q_{\gamma} f_{e}+Q_{\gamma-2} g_{\varepsilon}\right)\left[g_{\varepsilon}(x)-k\right]^{+} d x
\end{aligned}
$$


where we have introduced $Q_{\gamma}=P^{\gamma}-R_{2}^{\gamma}$ and $Q_{\gamma-2}=R_{2}^{\gamma}\left(\mu A \mu_{1}\right)-P_{\gamma-2}-R_{3}$ to keep the notation short. This proves the lemma

5.2 We prove an embedding theorem which is needed later in this section.

Lemma 3. Suppose $\Omega \subset \mathbb{R}^{N}$ is a domain and $s \in(0,1)$ is given. We set $\frac{1}{q}=\frac{1}{2}-\frac{s}{N}$, i.e. $q=\frac{2 N}{N-2 s}>2$. Then the following assertions are true.

1. We have the continuous embedding $W^{s, 2}(\Omega) \subset L_{q}(\Omega)$, such that

$$
\|u\|_{q} \leq c\|u\|_{s, 2} \quad \text { for all } u \in W^{s, 2}(\Omega) .
$$
that

2. If $\Omega_{1} \subset \subset \Omega$ is an open set, then there exists a constant $C=C\left(\Omega, \Omega_{1}\right)>.0$ such

$$
\|u\|_{q} \leq C|u|_{s, 2} \quad \text { for all } u \in W^{s, 2}(\Omega) \text { with } \operatorname{supp} u \subseteq \Omega_{1} .
$$

Proof. For Assertion 1 cf. Triebel [16: p. 196]. For Assertion 2,we prove that

$$
u \longmapsto\|u\|_{a}=\left\{|u|_{s, 2}^{2}+\int_{\Omega \backslash \Omega_{1}}|u|^{2}\right\}^{1 / 2}
$$

is an equivalent norm on $W^{s, 2}(\Omega)$, i.e. there exist constants $c_{1}, c_{2}>0$ such that

$$
c_{1}\left\{|u|_{s, 2}^{2}+\int_{\Omega \backslash \Omega_{1}}|u|^{2} d x\right\} \leq\left\{|u|_{s, 2}^{2}+\int_{\Omega}|u|^{2} d x\right\} \leq c_{2}\left\{|u|_{s, 2}^{2}+\int_{\Omega_{\backslash \Omega_{1}}}|u|^{2} d x\right\}
$$

for all $u \in W^{s, 2}(\Omega)$. The first inequality in (49) is obvious. To prove the second one we suppose the contrary. Then there exists an sequence $\left(u_{n}\right)_{n \in N}$ such that $\left\|u_{n}\right\|_{s, 2} \geq$ $n\left\|u_{n}\right\|_{a}(n \in \mathbb{N})$. We define $v_{n}=\frac{u_{n}}{\left\|u_{n}\right\|_{0,2}}$. Thus $\left\|v_{n}\right\|_{s, 2}=1$ and $\left\|v_{n}\right\|_{a} \rightarrow 0$, and we can select a subsequence, again denoted by $\left(v_{n}\right)$ such that $v_{n} \rightarrow v$ in $W^{s, 2}(\Omega), v_{n} \rightarrow v$ in $L_{2}(\Omega)$ and $v_{n}(x) \rightarrow v(x)$ a.e. in $\Omega$. From $\left\|v_{n}\right\|_{a} \rightarrow 0$ it follows that

$$
v_{n}(x) \rightarrow 0 \quad \text { a.e. in } \Omega \backslash \Omega_{1}
$$

and

$$
\left|v_{n}\right|_{s, 2}^{2}=\iint_{\Omega \times \Omega}|x-y|^{-N-2 s}\left|v_{n}(x)-v_{n}(y)\right|^{2} d x d y \rightarrow 0 .
$$

Therefore $\left|v_{n}(x)-v_{n}(y)\right| \rightarrow 0$ a.e. in $\Omega \times \Omega$ and (50) implies $v_{n}(x) \rightarrow 0$ a.e. in $\Omega$. This gives $v_{n} \rightarrow 0$ in $L_{2}(\Omega)$ and because of $\left|v_{n}\right|_{9,2} \rightarrow 0$ we see that $\left\|v_{n}\right\|_{9,2} \rightarrow 0$, which contradicts $\left\|v_{n}\right\|_{s, 2}=1$. Thus (49) is proved, and (47) follows immediately

5.3 Now we define sets $A_{\varepsilon}(k)$ where $g_{\ell}=\varphi u_{\epsilon}^{\delta}$ superceeds a level $k$ :

$$
A_{\varepsilon}(k)=\left\{x \in \Gamma: g_{\varepsilon} \geq k\right\} \text {. }
$$

We age going to estimate the size of $A_{\varepsilon}(k)$. Remember that $1<\gamma<2$. 
Lemma 4. We suppose $b \in W_{\text {loc }}^{\gamma, r}(\Gamma)$ for some $\gamma \in(1,2)$ and $r>\frac{N}{\gamma-1}$. Set $b_{e}:=$ $b^{\delta}:=b$. Then there exist constants $C>0$ and $\beta>1$, independent from $\varepsilon$ and $\delta$, such that

$$
\left|A_{\epsilon}(h)\right| \leq \frac{C}{(h-k)^{q}}\left|A_{e}(k)\right|^{\beta} \quad \text { for all } h>k \geq 0
$$

where $q=\frac{2 N}{N+1-\gamma}$.

Proof. Set $s=\frac{\gamma-1}{2}, q=\frac{2 N}{N-2 s}=\frac{2 N}{N+1-\gamma}>2$ and $\frac{1}{q}+\frac{1}{q^{\prime}}=1$. It follows from Lemma 2, Lemma 3 and the inclusion $\operatorname{supp}\left[g_{\varepsilon}(x)-k\right]^{+} \subseteq \operatorname{supp} \varphi \subset \subset U$ that

$$
\begin{aligned}
& \left\{\int_{A_{\varepsilon}(k)}\left|\left[g_{\varepsilon}(x)-k\right]^{+}\right|^{q} d x\right\}^{2 / q} \\
& \quad \leq c\left\{\int_{A_{\varepsilon}(k)}\left(\left|Q_{\gamma} f_{\varepsilon}\right|+\left|Q_{\gamma-2} g_{\varepsilon}\right|\right)^{q^{\prime}} d x\right\}^{1 / q^{\prime}}\left\{\int_{A_{\varepsilon}(k)}\left|\left[g_{\varepsilon}(x)-k\right]^{+}\right|^{q} d x\right\}^{1 / q}
\end{aligned}
$$

for $k \geq 0$. Young's inequality gives

$$
\left\{\int_{A_{\varepsilon}(k)}\left|\left[g_{e}(x)-k\right]^{+}\right|^{q} d x\right\}^{2 / q} \leq c\left\{\int_{A_{\varepsilon}(k)}\left(\left|Q_{\gamma} f_{\varepsilon}\right|+\left|Q_{\gamma-2} g_{\varepsilon}\right|\right)^{q^{\prime}} d x\right\}^{2 / q^{\prime}} .
$$

Therefore, for $h>k \geq 0$,

$$
\left|A_{e}(h)\right|(h-k)^{q} \leq c\left\{\int_{A_{\varepsilon}(k)}\left(\left|Q_{\gamma} f_{e}\right|+\left|Q_{\gamma-2} g_{e}\right|\right)^{q^{\prime}} d x\right\}^{q / q^{\prime}}
$$

and, by Hölder's inequality with $r>\frac{q}{q-2}=\frac{N}{\gamma-1}$ and $r>q^{\prime}$,

$$
\left|A_{\varepsilon}(h)\right|(h-k)^{q} \leq c\left(\left\|Q_{\gamma} f_{\varepsilon}\right\|_{r, U}+\left\|Q_{\gamma-2} g_{\varepsilon}\right\|_{r, U}\right)^{q}\left|A_{\varepsilon}(k)\right|^{q-1-\frac{q}{r}} .
$$

We see that $\beta=q-1-q>1$. It follows from (22) and (30) in the proof of Theorem 1 that $\sup \left(\left\|Q^{\gamma} f_{e}\right\|_{r, U}+\left\|Q_{\gamma-2} g_{e}\right\|_{r, U}\right)<+\infty$. This gives (51)

Now we are in the position to prove the uniform boundedness of the family $\left(u_{\varepsilon}\right)=$ $\left(u_{\varepsilon}^{\delta}\right)$. We are going to use the following result of Stampacchia.

Lemma 5 (see Kinderlehrer and Stampacchia [8: p. 63]). Let $\phi:\left[k_{0},+\infty\right) \rightarrow \mathbb{R}$ be a non-negative and non-increasing function such that

$$
\phi(h) \leq \frac{C}{(h-k)^{\alpha}}[\phi(k)]^{\beta} \quad \text { for } h>k>k_{0}
$$

where $C, \alpha$ and $\beta$ are positive constants with $\beta>1$. Then

$$
\phi\left(k_{0}+M\right)=0
$$


where

$$
M=2^{\frac{\rho}{\beta-1}} C^{\frac{1}{a}}\left[\phi\left(k_{0}\right)\right]^{\frac{\rho-1}{\alpha}} .
$$

Theorem 2. Suppose $b \in W^{\gamma, r}(U)$ for some $\gamma \in(1,2)$ and $r>\frac{N}{\gamma-1}$. Then the solution $u$ of the variational inequality (1) is locally bounded on $\Gamma: u \in L_{\infty}^{\text {loc }}(\Gamma)$, i.e. for all $V \subset \subset \Gamma$ there exists a constant $M>0$ such that $0 \leq u(x) \leq M$ a.e. on $V$.

Remark 2. Under the hypotheses of Remark 1 one may prove the inclusion $u \in$ $L_{\infty}(S)$.

Proof of Theorem 2. We shall prove the theorem in three steps.

(a) First we define $b_{\varepsilon}^{\delta}=b^{\delta}:=b$ for all $\varepsilon, \delta>0$. We are going to apply Lemma 5 and suppress the superscript $\delta$ again. Set $\phi_{\varepsilon}(k)=\left|A_{\varepsilon}(k)\right|$ and $k_{0}=0$. Then $\phi_{e}\left(k_{0}\right)=$ $\left|\left\{x \in \Gamma: g_{\varepsilon} \geq 0\right\}\right| \leq|U|$ and it follows from (51) that there exists a bound $M>0$ independent of $\varepsilon$ and $\delta$ such that

$$
\varphi(x) u_{\varepsilon}^{\delta}(x)=g_{\varepsilon}(x) \leq M:=\sup _{\varepsilon} 2^{\frac{\beta}{\beta-1}} C^{\frac{1}{q}}\left[\phi_{\varepsilon}(0)\right]^{\frac{\rho-1}{q}} \leq c_{1} \mid U^{\frac{\rho-1}{q}}
$$

a.e. on $U$.

(b) Next, we keep $\delta>0$ fixed and let $\varepsilon:=\varepsilon_{n} \rightarrow+0$. For simplicity, we omit the subscipt $n$. From Proposition 2 we know that $u_{e} \rightarrow u^{\delta}$ in $L_{2}(S), g_{\varepsilon} \rightarrow g^{\delta}=\varphi u^{\delta}$ in $L_{2}(U)$ and along a subsequence $g_{\varepsilon}(x) \rightarrow g^{\delta}(x)$ a.e. in $U$. Since $u^{\delta} \in K_{1}(56)$ gives

$$
0 \leq \varphi(x) u^{\delta}(x)=g^{\delta}(x) \leq M
$$

a.e. in $U$.

(c) Finally, let $\delta:=\delta_{n} \rightarrow+0$. As in the proof of Theorem 1 we have $\varphi u^{\delta} \rightarrow \varphi u$ in $L_{2}(U)$, and $\varphi u^{\delta} \rightarrow \varphi u$ in $W^{-\frac{1}{2}, 2}(S)$. Along a subsequence, a theorem of Banach and Saks (see Riesz and Sz.-Nagy [11: p.72]) implies the strong $L_{2}$-convergerice of the sequence of arithmetic means, i.e. $v_{n}=\frac{1}{n}\left(\varphi u^{\delta_{1}}+\varphi u^{\delta_{2}}+\ldots+\varphi u^{\delta_{n}}\right) \rightarrow \varphi u$ in $L_{2}(U)$. Again, passing to a subsequence if necessary, $v_{n}(x) \rightarrow \varphi(x) u(x)$ a.e. in $U$. Since for the means $0 \leq v_{n}(x) \leq M$ we have also $0 \leq \varphi(x) u(x) \leq M$ a.e. in $U$. As we may choose $\varphi$ in Subsection 4.1 such that $\varphi \equiv 1$ on an arbitrary open set $V \subset \subset U$ the assertion follows

\section{References}

[1] Adams, R.: Sobolev spaces. New York: Academic Press 1978.

[2] Archipova, A. A. and N. N. Uralzeva: On the regularity of the solution of a problem with one-sided obstacles on the boundary (in Russian). Vestnik Leningr. Univ. (Ser. 1) (1986)1, 3 - 10.

[3] Barbu, V. and Th. Precupanu: Convexity and Optimization in Banach Spaces. Leyden: Sijthoff \& Nordhoff 1978.

[4] Dieudonné, J.: Grundzüge der modernen Analysis, Vol. 7. Braunschweig: Vieweg 1982, and Berlin: Dt. Verlag Wiss. 1982. 
[5] Hörmander, L.: The Analysis of Linear Partial Differential Operators. Vols. 1 - 4. New York - Berlin: Springer-Verlag 1989 - 1992.

[6] Kinderlehrer, D.: The smothness of the solution of the boundary obstacle problem. J. Math. Pures Appl. 60 (1981), 193 - 212.

[7] Kindeilehrer, D.: Remarks about Signorini's problem in linear elasticity. Ann. Sci. Norm. Sup. Pisa 8 (1981), $605-645$.

[8] Kinderlehrer, D. and G. Stampacchia: An Introduction to Variational Inequalities and their Applications. New York: Academic Press 1980.

[9] Lions, J.L.: Quelques méthodes de résolution des problèmes aux limites non linéaires. Paris: Dunod 1969.

[10] Mund, A.: Equilibrium configurations for rotating conducting bodies. Univ. Leipzig (unpublished).

[11] Riesz; F. und B.Sz.-Nagy: Vorlesungen über Funktionalanalysis. Berlin: Dt. Verlag Wiss. 1973.

[12] Rodrigues, J. F.: Obstacle Problems in Mathematical Physics. Amsterdam: North Holland 1987.

[13] Schumann, R.: Regularity for Signorini's problem in linear elasticity. Man. math. 63 (1989), $255-291$.

[14] Shamir, E.: Regularity of mixed second order elliptic problems. Israel Math. J. 6 (1968), $.150-168$.

[15] Treves, F.: Introduction to Pseudodifferential and Fourier Operators. New York: Plenum Press 1982.

[16] Triebel, H.: Theory of Functions Spaces. Leipzig: Akad. Verlagsges. Geest \& Portig 1983 and Basel: Birkhäuser Verlag 1983.

[17] Uralzeva, N. N.: On the regularity of solutions of variational inqualities (in Russian). Uspechi mat. Nauk 42 (1987)6, 151 - 174.

[18] Wloka, J.: Partielle Differentialgleichungen. Stuttgart: Teubner-Verlag 1982.

[19] Zeidler, E.: Nonlinear Functional Analysis and Applications. Vols. 1 - 4: New YorkBerlin: Springer-Verlag 1985 - 1992. 